EPJ Web of Conferences 66, 07008 (2014)

DOI: $10.1051 /$ epjconf/ 20146607008

(C) Owned by the authors, published by EDP Sciences, 2014

\title{
Application of the Trojan Horse Method to study neutron induced reactions: the ${ }^{17} O(n, \alpha){ }^{14} \mathrm{C}$ reaction
}

M. Gulino ${ }^{1,2, a}$, C. Spitaleri ${ }^{1,3}$, X.D. Tang ${ }^{4}$, G.L. Guardo ${ }^{1,3}$, L. Lamia ${ }^{1,3}$, S. Cherubini ${ }^{1,3}$, B. Bucher $^{4}$, V. Burjan ${ }^{5}$, M. Couder ${ }^{4}$, P. Davies ${ }^{4}$, R. deBoer ${ }^{4}$, X. Fang ${ }^{4}$, V.Z. Goldberg ${ }^{6}$, Z. Hons ${ }^{5}$, V. Kroha ${ }^{5}$, L. Lamm ${ }^{4}$, M. La Cognata ${ }^{1}$, C. $\mathrm{Li}^{7}$, C. Ma ${ }^{4}$, J. Mrazek ${ }^{5}$, A.M. Mukhamedzhanov ${ }^{6}$, M. Notani ${ }^{4}$, S. O’Brien ${ }^{4}$, R.G. Pizzone ${ }^{1}$, G.G. Rapisarda ${ }^{1,3}$, D. Roberson ${ }^{4}$, M.L. Sergi ${ }^{1}$, W. $\mathrm{Tan}^{4}$, I.J. Thompson ${ }^{8}$, and M. Wiescher ${ }^{4}$

${ }^{1}$ INFN Laboratori Nazionali del Sud, Catania, Italy

${ }^{2}$ Università degli Studi di Enna "KORE", Enna, Italy

${ }^{3}$ Dipartimento di Fisica ed Astronomia, Università degli Studi di Catania, Catania, Italy

${ }^{4}$ Department of Physics and Joint Institute for Nuclear Astrophysics, University of Notre Dame, IN, USA

${ }^{5}$ Nuclear Physics Institute of ASCR, Rez, Czech Republic

${ }^{6}$ Cyclotron Institute, Texas A\&M University, College Station, TX, USA

${ }^{7}$ China Institute of Atomic Energy, Beijing, China

${ }^{8}$ Lawrence Livermore National Laboratory, Livermore, CA, USA

\begin{abstract}
The reaction ${ }^{17} \mathrm{O}(n, \alpha){ }^{14} \mathrm{C}$ was studied using virtual neutrons coming from the quasi-free deuteron break-up in the three body reaction ${ }^{17} \mathrm{O}+d \rightarrow \alpha+{ }^{14} \mathrm{C}+p$. This technique, called virtual neutron method, extends the Trojan Horse method to neutroninduced reactions allowing to study the reaction cross section avoiding the suppression effects coming from the penetrability of the centrifugal barrier. For incident neutron energies from thermal up to a few hundred $\mathrm{keV}$, direct experiments have shown the population of two out of three expected excited states at energies $8213 \mathrm{keV}$ and $8282 \mathrm{keV}$ and the influence of the sub-threshold level at $8038 \mathrm{keV}$. In the present experiment the ${ }^{18} \mathrm{O}$ excited state at $E^{*}=8.125 \mathrm{MeV}$, missing in the direct measurement, is observed. The angular distributions of the populated resonances have been measured for the first time. The results unambiguously indicate the ability of the method to overcome the centrifugal barrier suppression effect and to pick out the contribution of the bare nuclear interaction.
\end{abstract}

\section{Introduction}

The reaction ${ }^{17} \mathrm{O}(n, \alpha){ }^{14} \mathrm{C}$ is interesting for both nuclear energy and nuclear astrophysics: in nuclear reactors this reaction is one of the dominant sources of the long lasting radioactive isotope ${ }^{14} \mathrm{C}$ $\left(T_{1 / 2}=5730 \mathrm{yr}\right)$ [1]. In nuclear astrophysics, this reaction takes place in various astrophysical scenarios in the nucleosynthesis of heavier elements [2,3], and it could help to explain anomalies in ${ }^{18} \mathrm{O} /{ }^{16} \mathrm{O}$ and ${ }^{17} \mathrm{O} /{ }^{16} \mathrm{O}$ ratios found in asymptotic giant branch stars and in circumstellar $\mathrm{Al}_{2} \mathrm{O}_{3}$ meteorite grains [4]. For all these reasons, this reaction has been studied by several groups both using direct and indirect methods [5-8]. Direct experiments have shown the influence of the subthreshold level at 8038

\footnotetext{
ae-mail: gulino@lns.infn.it
}

This is an Open Access article distributed under the terms of the Creative Commons Attribution License 2.0, which permits unrestricted use, distribution, and reproduction in any medium, provided the original work is properly cited. 


\section{EPJ Web of Conferences}

Table 1. Details of the experimental set-up used in the LNS and NSL experiments

\begin{tabular}{lll}
\hline & LNS exp. & NSL exp. \\
\hline beam energy & $41 \mathrm{MeV}$ & $43.5 \mathrm{MeV}$ \\
target thickness & $150 \mu \mathrm{g} / \mathrm{cm}^{2}$ & $170 \mu \mathrm{g} / \mathrm{cm}^{2}$ \\
detectors angles & $5^{\circ}-10^{\circ} ; 13.3^{\circ}-20.8^{\circ} ; 21.5^{\circ}-29.0^{\circ}$ & $5^{\circ}-10^{\circ} ; 13.1^{\circ}-18.1^{\circ} ; 23.8^{\circ}-28.8^{\circ}$ \\
\hline
\end{tabular}

$\mathrm{keV}$ and the population of the two excited states at $8213 \mathrm{keV}$ and $8282 \mathrm{keV}$. No evidence is reported for the expected $8125 \mathrm{keV}$ state of ${ }^{18} \mathrm{O}\left(\mathrm{J}^{\pi}=5^{-}\right)$, because of the high angular momentum involved for this excited state $\left(l_{i}=3\right)$. However, the different data sets are not in agreement expecially at low energies, and the reaction rate calculated at the astrophysical relevant temperatures $\left(\mathrm{T} \in[0.01-1.3] \cdot 10^{9}\right.$ $\mathrm{K}$ ) differs by a factor 2-2.5 with a consequent change in the abundance ratios for some elements (e.g. ${ }^{22} \mathrm{Ne},{ }^{26} \mathrm{Mg}$ ) [2].

In the last decades, the Trojan Horse method (THM) [9-16] has been developed as an indirect method in order to study charged-particle induced reactions nuclear reactions, at the low energies of astrophysical interest. Recently, it has been extended to the indirect study of neutron induced reactions $[15,16]$. The principles of the method and its experimental applications are described in Ref. [9-16].

In the present work, we present the indirect study of the ${ }^{17} \mathrm{O}(n, \alpha){ }^{14} \mathrm{C}$ reaction performed by using the ${ }^{17} \mathrm{O}+d \rightarrow \alpha+{ }^{14} \mathrm{C}+p$ one, and selecting the events proceeding through the quasi-free (QF) breakup of the deuteron, following the THM prescriptions. As the THM is not affected by the orbital momentum barrier, the results provide observational evidence for resonance contributions from all known states. In the next sections the experiments, the data analysis and the achieved results are reported.

\section{The experiment}

The ${ }^{17} \mathrm{O}+d \rightarrow \alpha+{ }^{14} \mathrm{C}+p$ reaction has been measured in two different experimental runs: the first one was performed at the Laboratori Nazionali del Sud (LNS) in Catania, Italy, and the second one at the Nuclear Science Laboratory (NSL) of the University of Notre Dame, USA. In both the experiments a ${ }^{17} \mathrm{O}$ beam was impinging onto a $\mathrm{CD}_{2}$ target and the angles and energies of the ejectiles $\alpha$ and ${ }^{14} \mathrm{C}$ were detected in coincidence using an experimental set-up, symmetric with respect to $0^{\circ}$. The energy and angle of the third particle were reconstructed from kinematical calculations. The experimental set-up consists of 6 Position Sensitive Silicon Detectors (PSDs, single area, resistive readout, spatial resolution $0.5 \mathrm{~mm}$ ). The two most forward detectors were equipped with ionization chambers (ICs) used as $\Delta E$ detectors to identify carbon. Coincidences among one of the two forward PSD detectors and one of the three placed on the opposite side with respect to the beam axis were recorded by the data acquisition system. Details about the experimental set-up used for the two experiments are reported in table 1 .

\section{Data analysis and results}

The reaction of interest was identified by selecting events in which a carbon was detected in one of the telescopes. The reconstructed $Q$-value for the three-body reaction $(Q=-0.6 \pm 0.3 \mathrm{MeV})$ is in agreement with the expected one $(Q=-0.407 \mathrm{MeV})$. As a second step in the data analysis, the predominance of the QF reaction mechanism among other reaction processes filling the same final state has been checked by using several tests described elsewhere [9-15]. If the events having momentum of the proton spectator less than $50 \mathrm{MeV} / \mathrm{c}$ are considered, the obtained results are well reproduced 


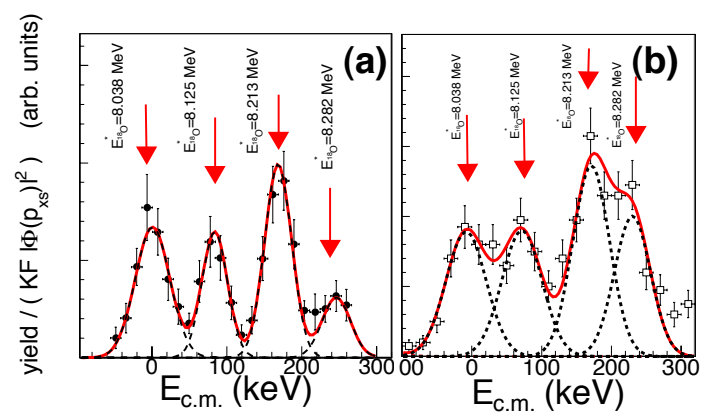

Figure 1. Excitation function of the ${ }^{17} \mathrm{O}(n, \alpha){ }^{14} \mathrm{C}$ reaction from LNS experiment for $\theta_{c m}=55^{\circ} \pm 5^{\circ}$ (a) and from NSL experiment for $\theta_{c m}=65^{\circ} \pm 25^{\circ}$ (b). The red solid line is a fit using four Gaussian functions; the black dashed lines represent their individual contributions.

by using both Distorted Wave Born Approximation (DWBA) and Plane wave Born Approximation (PWIA) approach. As no absolute values of the cross sections are extracted, the PWIA approach was used. In this approach, the cross section of the three body reaction can be factorized into two terms: the former describes the break-up of he deuteron and is calculated by a Monte Carlo simulation, the latter represents the cross section of the two-body reaction of interest. The cross section of the ${ }^{17} \mathrm{O}(n, \alpha){ }^{14} \mathrm{C}$ reaction was then extracted by dividing the experimental yield by the first term, and it is shown in fig. 1: panel (a) refers to the LNS experiment while panel (b) to the NSL one. The population of the four ${ }^{18} \mathrm{O}$ resonances expected in the explored energy region is evident. This result demontrates that the extracted two body cross section does not suffer from the centrifugal barrier penetration effect. The solid line in figure 1 is a fit performed using four gaussian functions to disentangle the contributions of the four ${ }^{18} \mathrm{O}$ resonances. No interference effect is taken into account in the fitting procedure, owing to the resonance widths $(<15 \mathrm{keV})$, which are much smaller than their energy separation. For the first time the angular distributions for the four resonances have been measured (data not shown, see Ref. [16]). The angular distribution of the resonance corresponding to the $E^{*}=8213 \mathrm{keV}$ level is better reproduced by adopting $l_{i}=2$ instead of $l_{i}=0$, as usually assumed by considering the low value of the neutron energy availabe in the c.m. system [5]. To make a comparison between the indirect and the direct measured cross section, the indirect data were multiplied for the penetrability of the centrifugal barrier calculated for each populated resonance. The comparison is shown in fig. 2, where the dotted line reports the direct data from Ref. [5], the solid line represents the same direct data spreaded out at the same resolution of the indirect ones and the data points are the indirect measured cross section.

\section{Conclusions}

The evidence of the population of the four ${ }^{18} \mathrm{O}$ resonances expected in the energy range explored by the experiment demonstrates the effectiveness of the THM to emphasize the mere nuclear interaction, avoiding even the centrifugal suppressions. Moreover, the good agreement between the direct and indirect measured data sets after the correction for the penerability of the centrifugal barrier gives evidence of the possibility to use the THM to get information about nuclear structure parameters of orbital momentum-suppressed neutron capture resonances, opening new perspectives in the use of the method for nuclear structure studies. A particularly interesting application is the study of neutron induced reactions on short-lived radioactive nuclei using inverse kinematics. 


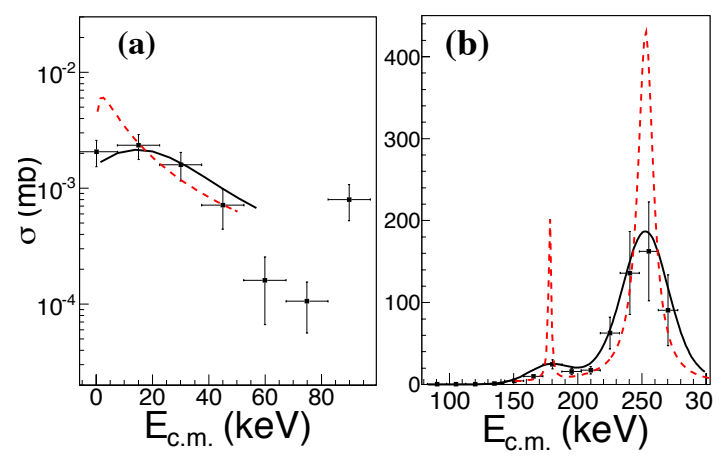

Figure 2. Cross section of the ${ }^{17} \mathrm{O}(n, \alpha){ }^{14} \mathrm{C}$ reaction from $\mathrm{TH}$ data after the correction for the penetrability of the centrifugal barrier (full dots) in the energy ranges 0-100 keV (a) and 100-300 keV (b). In both pannels, the dashed red line represents the direct data from Ref. [5], where available, while the solid line reports the direct data smeared at the TH energy resolution.

\section{Acknowledgments}

A.M. M. acknowledges the support by the US DOE under Grant Nos. DE-FG02-93ER40773, DEFG52-09NA29467 and DE-SC0004958 and NSF under Grant No. PHY-0852653. I.J. T. acknowledges the support by the US DOE by LLNL under Contract DE-AC52-07NA27344. V.B., Z.H., V.K. and J.M. acknowledge the support of the AMVIS Project under Grant No. M10480902 and LH11001 and of the GACR Project under Grant No. P203/10/0310. The Notre Dame collaborators were supported by the NSF under Grants No. PHY-0758100 and No. PHY-0822648.

\section{References}

[1] M-S Yim, F. Caron, Prog. Nucl. Ener. 48, 2 (2006)

[2] J. Applegate et al. Astroph.J. 329, 572 (1988)

[3] M. Forestini et al. Astron. Astroph. 123, 241 (1997)

[4] L.R.Nittler et al NPA621, 113c (1997)

[5] J. Wagemans et al. Phys. Rev. C65(3), 034614 (2002)

[6] H. Schatz et al. Astroph. J. 413, 750 (1993)

[7] P.E. Koehler, S.M. Graff Phys. Rev. C44, 2788 (1991)

[8] R.M. Sanders, Phys. Rev. 104, 1434 (1956)

[9] S. Cherubini at al. ApJ 457, 855 (1996)

[10] C. Spitaleri et al., Nucl. Phys. A 71999 (2003)

[11] A. Tumino et al., Phys. Rev. Lett.98, 252502 (2007)

[12] M.L.Sergi et al., Phys. Rev. C82, 032801 (2010)

[13] M. La Cognata et al., Astroph. J. 708, 796 (2010)

[14] L. Lamia et al., J. Phys. G: Nucl.Part.Phys. 39, 015106 (2012)

[15] M. Gulino et al., J. Phys. G: Nucl.Part.Phys. 37, 125105 (2010)

[16] M. Gulino et al.,Phys. Rev. C 87, 012801(R) (2013) 